\title{
Meta-Analysis for Penalized Regression Methods with Multi-Cohort Genome-Wide Association Studies
}

\author{
Chen Lu ${ }^{a}$ George T. O'Connor ${ }^{b, c}$ Josée Dupuis ${ }^{a, c, d}$ Eric D. Kolaczyk ${ }^{d, e}$ \\ ${ }^{a}$ Department of Biostatistics, Boston University School of Public Health, and ${ }^{b}$ Pulmonary Center, Department of \\ Medicine, Boston University School of Medicine, Boston, Mass., 'The NHLBI's Framingham Heart Study, \\ Framingham, Mass., d Program in Bioinformatics, and e Department of Mathematics and Statistics, Boston University, \\ Boston, Mass., USA
}

\section{Key Words}

Meta-analysis · Multi-cohorts · Penalized regression · Data splitting

\begin{abstract}
Objective: Penalized regression has been successfully applied in genome-wide association studies. While meta-analysis is often conducted to increase power and protect patients' confidentiality, methods for meta-analyzing results of penalized regression in multi-cohort setting are still under development. Methods: We propose to use a data-splitting method to obtain valid $p$ values (or equivalently, coefficient estimates and standard errors) for meta-analysis across multiple cohorts. We examine two ways of splitting data in multicohort setting and propose three methods to conduct metaanalysis based on $p$ values. We compare the three metaanalysis methods to mega-analysis, which consists of pooling individual level data. We also apply our proposed meta-analysis approaches to the Framingham Heart Study data, where we divide the original dataset into four parts to create a multi-cohort scenario. Results: The simulations suggest that splitting cohorts has better performance than splitting data within each cohort. The real data application also shows that this method provides results that are similar to the megaanalysis. Conclusion: After comparing the three methods
\end{abstract}

that we proposed to conduct meta-analysis, we recommend splitting cohorts rather than datasets to obtain valid $p$ values for meta-analysis of results from penalized regression in multi-cohort setting.

(c) 2016 S. Karger AG, Basel

\section{Introduction}

Meta-analysis is a general approach to combine results from multiple cohorts and is routinely used in genomewide association studies (GWAS). To increase power to detect true signals, multiple studies are combined to increase sample size. Oftentimes, individual level data cannot be pooled among studies because of restrictions due to participants' confidentiality, so meta-analysis approaches are routinely used to combine summary statistics across studies, and this has been shown to be as powerful as merging individual datasets [1]. Widely used meta-analysis approaches include Fisher's method [2, 3], Stouffer's Z-score method [4] and inverse variance method using a fixed effect model $[5,6]$. These methods require valid $\mathrm{p}$ values for the regression coefficient $\beta$ or

Josée Dupuis and Eric D. Kolaczyk should be regarded as joint last authors.

\section{KARGER}

(c) 2016 S. Karger AG, Basel

E-Mail karger@karger.com

www.karger.com/hhe 
standard error (SE) of the estimates from all participating studies.

Penalized regression is an effective multivariate approach to select important predictors in GWAS [7, 8]. However, this method does not provide $\mathrm{p}$ values, because the regression estimates $(\beta)$ are shrunk due to the penalty involved in optimization and the fact that the $\beta$ s are used for variable selection (depending on whether the term being considered has a non-zero coefficient estimate or not) instead of effect size estimation $[9,10]$.

To apply penalized regression in a multi-cohort setting, two issues need to be addressed. The first is to obtain valid $\mathrm{p}$ values. Ordinary least squares are often used to obtain $\mathrm{p}$ values or effect size estimation $(\beta)$. But regular regression with selected terms cannot be applied to the same set of data that has been used for variable selection. Recent articles have focused on data-splitting methods to obtain $\mathrm{p}$ values for high-dimensional data. Wasserman and Roeder [10] proposed splitting the observations into two subsets, using one subset for variable selection and using the other subset to obtain valid p values. Meinshausen et al. [9] further suggested multiple splits instead of a single split, because the result of a single split highly depends on the arbitrary split. We employ a data-splitting method to obtain $p$ values in penalized regression.

The second challenge is to develop an appropriate procedure to meta-analyze across cohorts. We propose two extensions to the splitting method for meta-analysis: splitting within cohort and splitting cohorts. The first approach involves splitting of data for each cohort, followed by selection of variables on one subset and computation of $p$ values on the other subset, and with meta-analysis across all cohorts. This is a natural extension of the datasplitting method to multiple cohorts. The second approach involves splitting cohorts instead. Cohorts are split into two groups, one group used for variable selection and the other used for obtaining $p$ values and metaanalysis. This is a more practical approach because it simplifies the analysis burden from analysts for each study and reduces the possibility of making errors.

The rest of this article is organized as follows: first, we describe the methods we proposed based on two extensions of the data-splitting method. Then, we describe our simulation design and illustrate the application of our method using a real data from the Framingham Heart Study (FHS). After that, we show the result of a simulation study, compare the performance of different methods proposed and make recommendations based on the simulation results. In the result section, we also present results from our real data application.

Meta-Analysis for Penalized Regression Methods with Multi-Cohort GWAS

\section{Methods}

Obtaining p Values for Penalized Regression

Penalized regression has been widely used as variable selection procedure in high dimensions. Besides the classical Lasso [11], there are many alternatives and extensions [7, 12-18]. For the following four meta-analysis procedures, we assume that the same variable selection approach has been used in each cohort. In our simulation, we used the Lasso method implemented in the software Mendel [19]. However, the procedures we propose here for meta-analysis are applicable, in principle, to general penalized regression methods.

Let $Y$ be a quantitative trait of interest and $\left\{X_{j}\right\}_{j=1}^{p}$ be $p$ predictors representing single nucleotide polymorphisms (SNPs). To select important predictors in one study, we are interested in a model of the form

$$
Y=\beta_{0}+\sum_{j=1}^{p} \beta_{j} \mathbf{X}_{j}+\epsilon,
$$

where $\beta$ is estimated through a penalized least-squares criterion.

$$
\tilde{\beta}=\operatorname{argmin}_{\beta} \frac{1}{2}\left\|\mathbf{Y}-\sum_{j=1}^{p} \beta_{j} \mathbf{X}_{j}\right\|^{2}+P(\beta) .
$$

$P$ is a function of the coefficient vector $\beta$. The theoretical justification for penalized regression with the classical Lasso is well documented $[9,10]$.

To represent SNPs, the variables $X_{j}$ are usually coded as the number of minor alleles $(0,1,2)$. The goal of the variable selection method is to identify a small set of SNPs associated with the dependent variable $Y$. After variable selection, we estimate $p$ values (or equivalently $\beta$ and SE) using one of the data-splitting methods described in the next section.

As mentioned earlier, there are several different ways to conduct meta-analysis in a regular regression framework. Fisher's method $[2,3]$ provides a way of combining $\mathrm{p}$ values across studies, but it does not take into account the direction of the effects. Stouffer's Z-score method [4] solved this problem by using signed $Z$-scores instead of $\mathrm{p}$ values. To account for directionality of effect in the following four procedures, we obtain Z-scores instead of $\mathrm{p}$ values.

Unlike Fisher's method and Stouffer's Z-score method, the inverse variance-based method $[5,6]$ estimates $\beta$ coefficient (effect sizes) and SE in addition to Z-scores (or equivalently p values). But not all of the procedures can utilize this method depending on the different data-splitting scheme. So we obtain $\beta$ and SE when available (Z-scores otherwise) from the data-splitting and regular regression steps, and use either the $Z$-scores or $p$ values as input for meta-analysis (inverse variance-based method when $\beta$ and SE are available, Stouffer's Z-score method otherwise). The result of the meta-analysis will be a set of $\beta$ and SE (or Z-scores) for the selected terms in the variable selection step $(\beta=0$ or $Z=0$ for terms not selected). One may also obtain p values from the final result of the meta-analysis. But in this article, we present the result in Z-scores to preserve directionality of effect estimates. Variations on our data-splitting approach are described in the following four procedures. 
Mega-Analysis: Combine All Data and Perform a Joint

Analysis

Procedure A: Mega-Analysis

When analyzing data from multiple studies involved in a consortium, if the studies are homogeneous, the ideal strategy would be to pool individual data together and conduct analysis as one dataset. Although not practical, it is a 'gold standard' when comparing to other procedures. Our goal is to find the meta-analysis procedure that behaves most similarly to the mega-analysis. We use the following algorithm to conduct Procedure A, the megaanalysis:

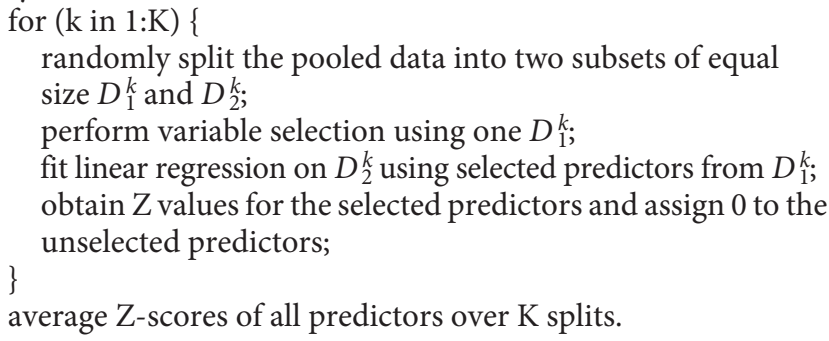

Proposed Meta-Analysis Approaches Based on Data-Splitting Principles

Procedure B: Split Cohorts into Two Groups

The data-splitting method $[9,10]$ suggests splitting the observations into two equal subsets, selecting variables using one subset and obtaining valid $\mathrm{p}$ values using the other subset. A practical extension of this method to multi-cohort setting is to split the cohorts into two groups. We select variables using cohorts in the first group, then calculate scores and conduct meta-analysis using cohorts in the second group. This is a two-step procedure. In the first step, all cohorts perform the variable selection procedure and provide a list of the selected predictors. Then we randomize the cohorts into two groups: groups 1 and 2. The splitting is performed $K$ times (where $K$ is the number of splits). For each data split, a list of predictors selected by any of the cohorts assigned to group 1 is generated, producing $K$ 'union' lists of selected variables. In the second step, cohorts are asked to fit at most $K$ final models, depending on the number of times they were assigned to group 2 during the cohort-splitting step.

This approach makes it easier for individual studies to perform the necessary analyses, and simplify the implementation of this approach within cohort. The algorithm for Procedure B is described below.

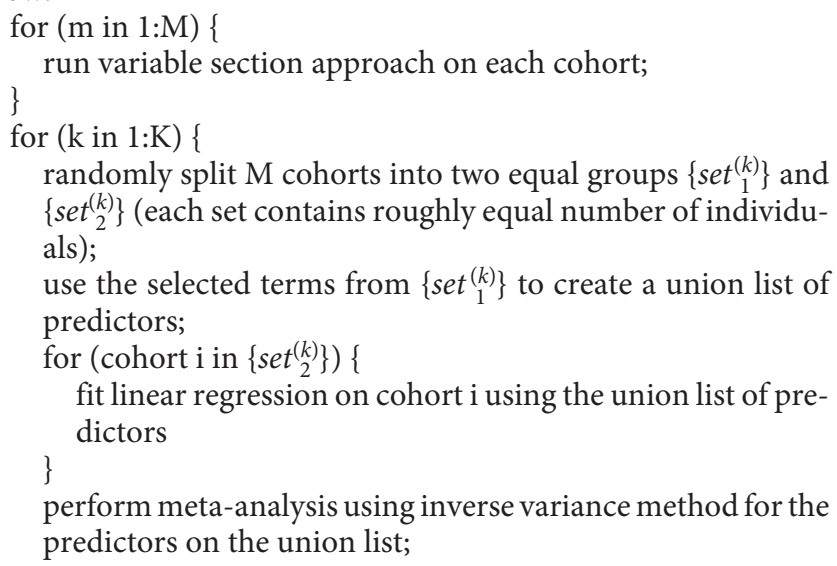

obtain Z-score for predictors on the union list and assign 0 for unselected predictors;

average Z-scores of all predictors over K splits.

Procedure C: Split Each Cohort into Two Parts

A natural extension of the data splitting method is to split data for each cohort and perform meta-analysis using results from each cohort. The following algorithm describes this approach.

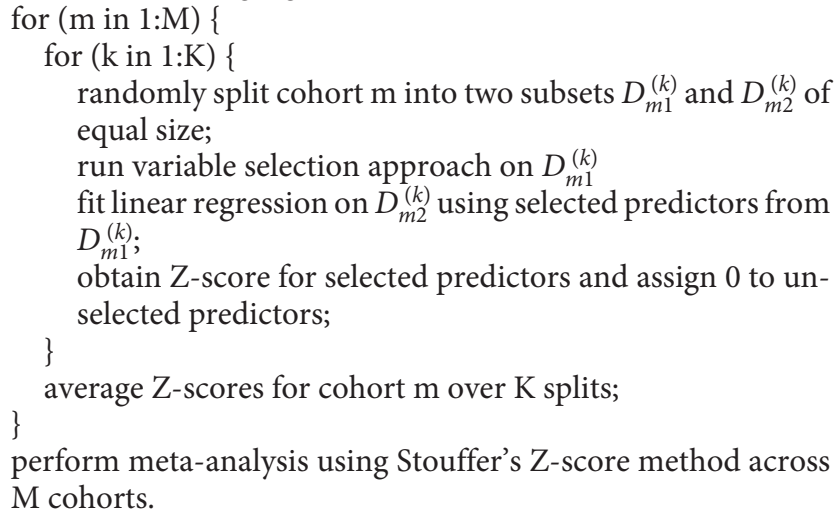

perform meta-analysis using Stouffer's Z-score method across $\mathrm{M}$ cohorts.

Procedure D: A Variation of Procedure C

This approach is a variation of Procedure C. In procedure C, each study conducts analysis (variable selection and $p$ values/ $Z$ value computation) separately without shared information among cohorts. The information is aggregated in the last step, the metaanalysis. It is possible and highly likely that the selected predictors are different among studies. Non-selected predictors are assigned zero Z-scores (or equivalently, a p value of 1 ). While in Procedure $\mathrm{B}$, there is shared information before obtaining $\mathrm{p}$ values. The list of selected terms is the union of all selected variables in the first group. To see if the sharing of information makes a difference, we modify Procedure $\mathrm{C}$ so that there is information sharing before obtaining $\mathrm{p}$ values. We name the modified version Procedure D.

This procedure is even less practical than Procedure $\mathrm{C}$ and increases the challenge in the sharing of results among studies. The main purpose of examining this procedure is to find out if the information sharing makes Procedure $\mathrm{C}$ perform differently from Procedures $\mathrm{A}$ and $\mathrm{B}$. The algorithm for Procedure $\mathrm{D}$ is described below.

for $(\mathrm{k}$ in $1: \mathrm{K})$ \{

for $(\mathrm{m}$ in $1: \mathrm{M})\{$ randomly split cohort $m$ into two subsets $D_{m 1}^{(k)}$ and $D_{m 2}^{(k)}$ of equal size;

\} run variable selection on $D_{m 1}^{(k)}$;

create union list of selected predictors from $D_{m 1}^{(k)}(\mathrm{m}=1, \ldots$, $\mathrm{M})$;

for $(\mathrm{m}$ in $1: \mathrm{M})\{$

fit linear regression on $D_{m 2}^{(k)}$ using the union list of select\} ed predictors

perform meta-analysis using inverse variance method for the predictors on the union list;

obtain Z-score for predictors on the union list and assign 0 \} for unselected predictors;

average Z-scores over K splits. 


\section{Simulation Design}

We conduct a simulation study to compare the performance of the three meta-analysis procedures and mega-analysis. Pooling samples (mega-analysis) is the ideal, but often infeasible, scenario. So our goal is to find the procedure that has similar performance to mega-analysis.

In our multi-cohort simulation, there are $M=10$ cohorts. Each dataset (cohort) has 1,000 subjects and 1,000 SNPs as predictors. The SNPs are coded additively $(0,1,2)$, simulated with a minor allele frequency of $50 \%$, and drawn from a binomial distribution with two trials. The quantitative trait is then simulated using the effect SNPs under the same model for all cohorts. The effect sizes are set for $80 \%$ power with a type 1 error rate of $5 \%$ under standard single-SNP additive models. Among the 1,000 SNPs, 20 (SNP1SNP20) have an effect on the simulated trait, and the proportion of variance explained by a SNP is 0.00845 . The remaining 980 SNPs have no effect.

In addition to the effect sizes corresponding to $80 \%$ power (strong), we also performed simulations where the effect sizes correspond to $50 \%$ power (moderate) and $20 \%$ power (weak) for sample size $n=1,000$ for each cohort, to evaluate the performance of the procedures under different effect sizes. To evaluate the effect of different sample sizes, we performed simulations with combinations of the three effect sizes (strong, moderate and weak) and two additional sample sizes ( $n=500$ and 2,000 for each cohort).

We also conducted simulation to test the performance of the procedures for cohorts with unequal sample sizes. Sample sizes in each of the 10 cohorts are 2,500, 2,000, 1,000, 1,000, 750, 750, 500, 500,500 and 500, respectively. The overall total sample size is the same as the simulation with equally sized cohorts described above, and the SNPs and quantitative trait Y are simulated in the same way, with 20 SNPs with a true effect on trait Y, while 980 SNPs have no effect.

We used the Lasso approach implemented in software Mendel [19] to perform variable selection in the three meta-analysis procedures and the mega-analysis. In the Mendel Lasso implementation, the tuning parameter is determined by pre-specifying a desired number of selected predictors. We set the tuning parameter so that 25 predictors are selected - slightly larger than the number of true effect SNPs (i.e., 20). Following the algorithms described above, we performed mega-analysis according to Procedure A and meta-analyses according to Procedures B-D. For multi-split, we used $K=10$, and for single split, we used $K=1$.

\section{Real Data Application}

Plasma IgE concentration is a biomarker that is often elevated in individuals with allergy to environmental allergens. Elevated plasma IgE concentration is associated with allergic diseases, including asthma, allergic rhinoconjunctivitis, atopic dermatitis and food allergy. A GWAS has been conducted on IgE concentration using data from the FHS [20]. Participants from the town of Framingham, Mass., have been recruited in this study starting in 1948, and have been followed over the years for the development of heart disease and related traits, including pulmonary function and allergic response measured by IgE concentration.

The dataset includes 6,975 participants, 441 from the original cohort recruited in 1948, 2,848 from the Offspring cohort recruited in 1971 and 3,686 from the Third Generation cohort initiated in 2002. There are 3,209 men and 3,766 women, aged 19 years and older. We analyze the natural logarithm of plasma total IgE con-

Meta-Analysis for Penalized Regression

Methods with Multi-Cohort GWAS centrations as our phenotype, adjusted for smoking (current, former and amount of life time smoking in terms of pack-years), age, sex and cohort of origin. Genotypes were from Affymetrix $500 \mathrm{~K}$ and MIPS 50K arrays, with imputation performed using the HapMap 2 European reference panel [21]. For high-dimensional data with correlated predictors (i.e. SNPs), the Lasso method tends to select one out of a group of correlated predictors. We used PLINK to prune SNPs based on linkage disequilibrium (LD) so that the SNPs in the final dataset have low LD (correlation). We used $r^{2}$ (pairwise genotypic correlation squared) of 0.8 as the threshold of LD pruning, removing one of a pair of SNPs in LD with $\mathrm{r}^{2}>0.8$. As a result, we have 659,236 SNPs in the final dataset (a subset of the 2.5 million SNPs used in the analysis in Granada et al. [20].

To apply our meta-analysis procedures, we created a 'pseudo multi-cohort' scenario using the data from the FHS. We divide the 6,975 subjects into 4 cohorts, 3 cohorts with 1,744 participants and 1 cohort with 1,743 participants. To create independent cohorts, all participants from a family were assigned to the same cohort.

We applied the mega-analysis Procedure A and the three proposed meta-analysis Procedures B-D to the 4 cohorts we created from the FHS data.

\section{Results}

\section{Simulation Results}

The simulation results are summarized in terms of values for all predictors averaged over 100 simulation replicates. As shown in figure 1, in the left panel are the values for active SNPs (average Z-scores of SNP1-SNP20) using procedures $\mathrm{A}, \mathrm{B}, \mathrm{C}$ and $\mathrm{D}$, respectively. In the right panel are the values for non-active SNPs (average Z-scores of SNP21-SNP1,000) using procedures A, B, C and D, respectively. Active SNPs of Procedure A (mega-analysis) have the highest average values, all greater than 6 . Procedure B (splitting cohorts into two groups) has the second highest average values. Procedure $\mathrm{C}$ (splitting each cohort into two subsets) has the lowest average $\mathrm{Z}$ values (close to 0 ). By modifying Procedure $\mathrm{C}$ to share information before obtaining valid $\mathrm{p}$ values, Procedure $\mathrm{D}$ has a better performance than $\mathrm{C}$, but substantially worse than $\mathrm{B}$.

We also tested all the procedures using single split $(K=1)$. The results of $Z$-scores averaged over 100 simulations are shown in figure 2. It is evident, the observations from figure 1 also hold. Procedure B is the one that performs most closely to mega-analysis, which should be expected if data of all cohorts were merged together and analyzed as one dataset. Also, the result of Procedure $\mathrm{C}$ is very different from mega-analysis. This difference reflects the same pattern we observe in the multiple splits. And by adding information sharing in Procedure C (i.e., Procedure D), it performs much more closely to mega-analysis, although not as close as Procedure B. 
Fig. 1. Simulation results for a multi-splitting method $(K=10)$ : average $Z$ values for SNPs using Procedures A-D.
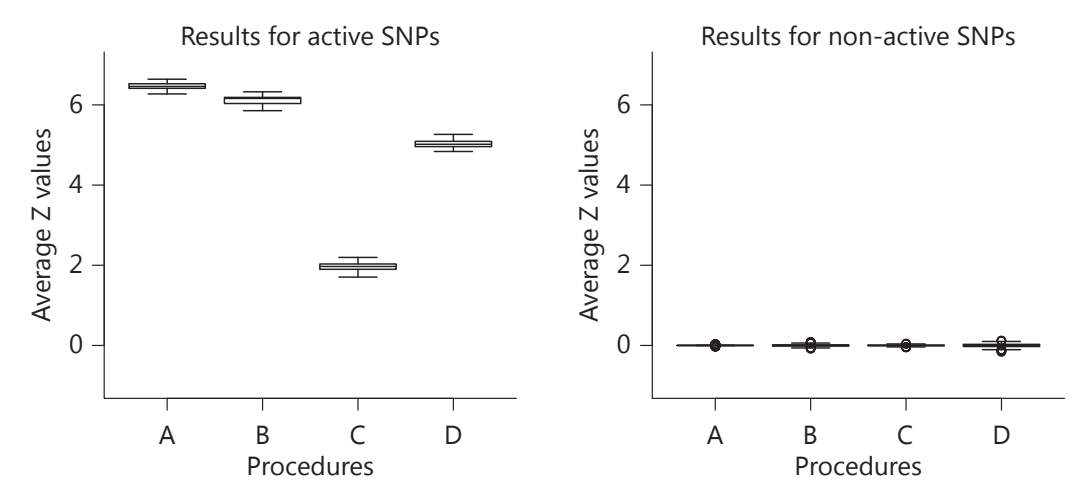

Fig. 2. Simulation results for a single-splitting method $(K=1)$ : average $Z$ values for SNPs using Procedures A-D.
Fig. 3. Simulation results for a multi-splitting method $(K=10)$ : standard error of $Z$ values for SNPs using Procedures A-D.
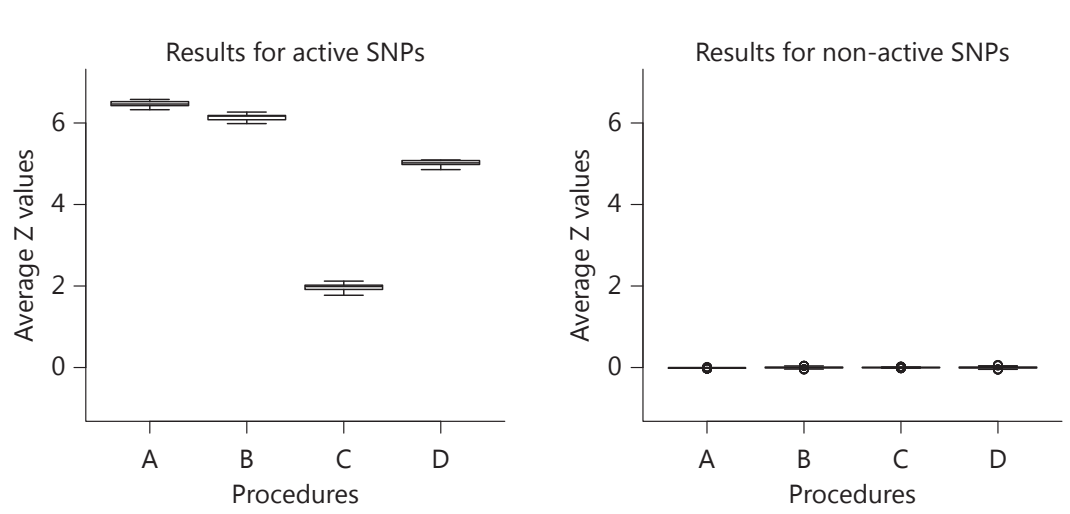
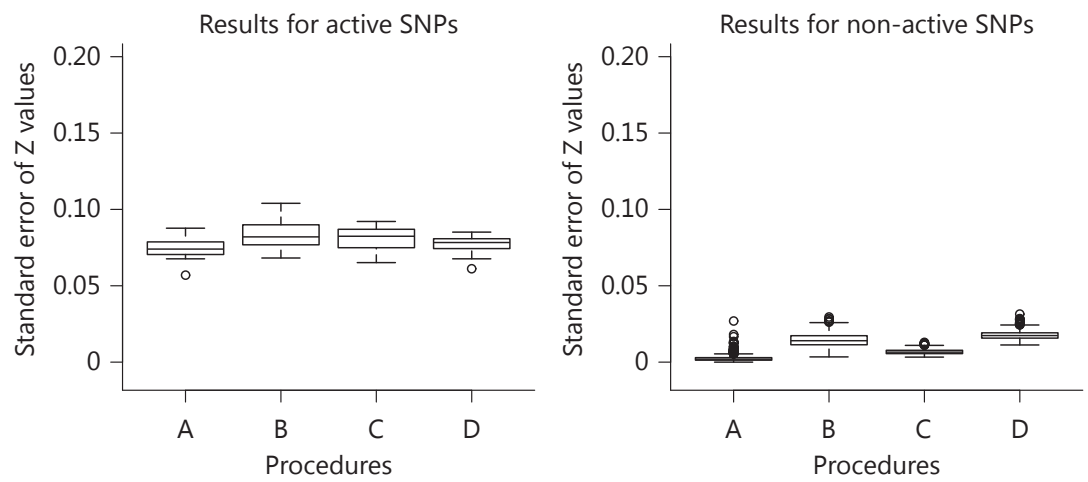
Fig. 4. Simulation results for a single-splitting method $(K=1)$ : standard error of $Z$ values for SNPs using Procedures A-D.

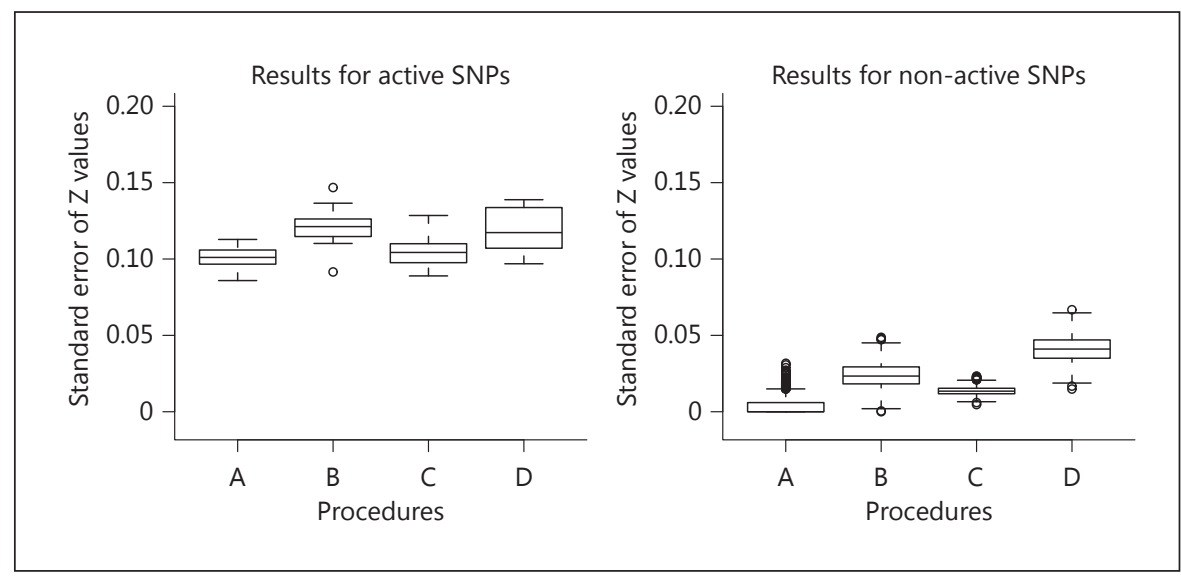

When comparing single split versus multiple splits, we examine the standard errors of the Z-scores. As Meinshausen et al. [9] pointed out, the multi-split method is better than single split because the result of the singlesplit method depends on the arbitrary split chosen. We present the standard errors of the Z-scores for multiple splits in figure 3 and those of single split in figure 4 . When comparing figure 3 and 4 , one can see that single split has larger standard errors of the Z-scores compared to multiple splits, indicating that the result of single split is more variable, which is consistent with what Meinshausen et al. [9] suggested.

The simulation results for unequally sized cohorts are shown in figure 5 (average Z-scores for both multiple splits and single split) and figure 6 (standard errors of the Z-scores). Similar to the simulation results with equally sized cohorts, Procedure B outperforms Procedures $\mathrm{C}$ and $\mathrm{D}$ in both multi-split and single-split methods, and the single-split approach has larger standard errors of the Z-scores compared to the multi-split approach.

The simulation results for moderate and weak effect sizes (sample size $n=1,000$ in each cohort) are shown in online supplementary figures 1 and 2 (for all online suppl. material, see www.karger.com/doi/10.1159/000447969). The simulation results for combinations of different effect sizes (strong, moderate and weak) and additionally two different sample sizes ( $n=500$ and 2,000 for each cohort) are shown in online supplementary figures 3-8. These results show that the performance of all procedures is consistent under all scenarios: Procedure B outperforms Procedures $\mathrm{C}$ and $\mathrm{D}$, and also performs most closely to Procedure A.

Meta-Analysis for Penalized Regression Methods with Multi-Cohort GWAS

\section{Real Data Application}

We applied the mega-analysis procedure (Procedure A) and the proposed meta-analysis procedures (Procedures $B, C$ and $D$ ) to the 'pseudo multi-cohort' created using data from the FHS. We used the R code that was developed earlier in our previous work [7] to perform variable selection in each of the proposed meta-analysis procedures, because imputed dosage genotype data in the real data is not supported by the Mendel binary SNP file format. We chose to look for 100 predictors in this variable selection step using the Lasso method. In the modelfitting step, we used a linear mixed-effect model to account for the correlation of subjects introduced by family structure.

The results of the analyses are shown in table 1. Values are ranked based on absolute values. Genes previously found in a GWAS of logarithm-transformed IgE levels in FHS participants [20] are indicated with an asterisk in the table. Because we chose to look for 100 predictors in the variable selection step, the final results of meta-analysis have more than 100 SNPs with non-zero values. We present the top $20 \mathrm{SNPs} /$ genes in the table. As shown in table 1, using Procedure B we found 2 out of 7 genes that were previously identified in a GWAS with a much larger sample size [20]. And one of the two genes is FCER1A (rs2251746), the strongest genetic variant associated with IgE concentration, as previously identified in Li et al. [20]. Procedures $\mathrm{C}$ and $\mathrm{D}$ found 1 previously identified gene, indicated with an asterisk in table 1. 
Table 1. Result of real data application: $Z$ values are ranked based on absolute value for the top 20 predictors selected

\begin{tabular}{|c|c|c|c|c|c|c|c|c|c|c|c|}
\hline \multicolumn{3}{|c|}{ Mega-analysis Procedure A } & \multicolumn{3}{|c|}{ Meta-analysis Procedure B } & \multicolumn{3}{|c|}{ Meta-analysis Procedure C } & \multicolumn{3}{|c|}{ Meta-analysis Procedure D } \\
\hline SNP & gene & $\mathrm{T}$ & SNP & gene & $\mathrm{Z}$ & SNP & gene & $\mathrm{Z}$ & SNP & gene & $\mathrm{Z}$ \\
\hline rs1801275 & IL4R & -4.54 & rs2251746 & FCER1A* & -3.61 & rs1868518 & CACNA2D3 & 1.11 & rs12438147 & TLN2 & 1.40 \\
\hline rs9548095 & TRPC4 & 4.42 & rs10870648 & ALOX5AP & 1.24 & rs8109578 & & 1.01 & rs92774458 & HLA-DPB1 & -1.17 \\
\hline rs17469756 & EMID2 & -4.14 & rs10485590 & & -1.17 & rs2071991 & AJAP1 & 0.89 & rs8109578 & & 1.02 \\
\hline rs4873193 & PXDNL & -4.07 & rs10797524 & & 1.16 & rs7176872 & & 0.74 & rs 2508050 & HLA-G* & 0.96 \\
\hline rs10951014 & & -4.03 & rs2360675 & KLF7 & -1.10 & rs8045887 & ZNF598 & -0.73 & rs6854454 & & 0.90 \\
\hline rs964626 & GABRR2 & -3.97 & rs17469756 & EMID2 & -1.08 & rs2055668 & & -0.66 & rs565433 & CAPN13 & 0.87 \\
\hline rs9920962 & RORA & -3.84 & rs2508050 & HLA-G* & 1.06 & rs9277458 & HLA-DPB1 & -0.65 & rs2031079 & ANKS4B & 0.86 \\
\hline rs12414220 & & 3.84 & rs719535 & ASTN2 & 1.04 & rs9657638 & PTPRD & 0.63 & rs9973132 & & 0.85 \\
\hline rs10485590 & & -3.80 & rs133992 & & 1.04 & rs 12250242 & & 0.61 & rs11735162 & KCNIP4 & 0.84 \\
\hline rs2774446 & TUBA3C & 3.77 & rs917605 & & -1.03 & rs10770224 & & -0.61 & rs2803443 & RFK & 0.81 \\
\hline rs12438147 & TLN2 & 3.75 & rs6904594 & & 0.96 & rs10891290 & PPP2R1B & 0.61 & rs12430009 & & 0.79 \\
\hline rs11646632 & SRL & 3.74 & rs7773053 & & -0.96 & rs 12636422 & & -0.59 & rs2124464 & PKNOX1 & 0.73 \\
\hline rs7042528 & ABL1 & -3.71 & rs12660817 & HLA-B & -0.93 & rs 2508050 & HLA-G* & 0.59 & rs833737 & & 0.72 \\
\hline rs4733828 & & -3.66 & rs7290574 & APOL3 & 0.92 & rs980895 & & 0.59 & rs16963045 & & -0.71 \\
\hline rs17208431 & MPP6 & 3.60 & rs2155219 & C11orf30 & 0.92 & rs917605 & & 0.56 & rs17081524 & DOK6 & -0.70 \\
\hline rs2572439 & & 3.58 & rs549196 & & 0.89 & rs2031079 & ANKS4B & 0.54 & rs16907813 & ELAVL2 & 0.67 \\
\hline rs9922602 & & -3.50 & rs6788832 & ST6GAL1 & -0.88 & rs38927 & ZNF804B & 0.53 & rs4533297 & & -0.67 \\
\hline rs6996341 & & -3.50 & rs152088 & ITGA2 & 0.88 & rs9527849 & & -0.53 & rs1868518 & CACNA2D3 & 0.65 \\
\hline rs1059513 & STAT6* & -3.44 & rs11715539 & LMCD1 & -0.86 & rs7109738 & & -0.52 & rs 12565755 & & -0.62 \\
\hline rs17704277 & HS3ST4 & -3.40 & rs10829691 & GLRX3 & 0.86 & rs12546438 & & -0.51 & rs998763 & & 0.62 \\
\hline
\end{tabular}

Asterisk indicates previously identified genes.

\section{Discussion}

We examined four procedures to conduct meta-analysis using penalized regression. The simulations showed that Procedure B is the one that performs most closely to the ideal case (i.e., when data are merged together and analyzed as one dataset). Modifying Procedure $\mathrm{C}$ to allow information sharing improves its performance to be closer to Procedure A, but still not as close as Procedure B is.

We compared the multi-split and single-split methods in simulations. Both of them showed that Procedure B has the most similar performance to Procedure A, compared to the other two procedures. But the multi-split method is clearly better than the single-split approach, in terms of the stability of the result.

Another interesting conclusion from our simulation study is that Procedure B is the preferred procedure in terms of performance, but it is also the most practical strategy among the three Procedures B, C and D. Although we have already shown that multiple splits are better than a single split, it is a complicated process to implement multiple splits into Procedures C and D. In Procedures $\mathrm{C}$ and $\mathrm{D}$, each cohort has to split their own data $K$ times, where $K$ is the number of splits. The variable selection and $\mathrm{Z}$ value estimation are done within each cohort. It will be very complicated to coordinate among studies, and this is an error-prone process. On the other hand, it is much easier to implement multiple splits in Procedure B. All cohorts perform the variable selection analysis only once. The $K$ random data splittings are under the control of the person performing the meta-analysis. According to the $K$ cohort assignments, the results of the cohorts in the first group are pooled and used to create $K$ lists of selected terms. The $K$ lists are sent to the cohorts in the second group, and these cohorts undergo a final model fitting. Each cohort will have to undergo the final fitting at most $K$ times.

Heterogeneity of genetic effect sizes might affect the ability of our approach to identify variants influencing the trait of interest. Accounting for heterogeneity would require an extension of our method possibly by using a different approach to meta-analyze results from the second stage, such as a random-effects approach or a transethnic meta-analysis as implemented in the software MANTRA [22]. Further investigation is needed on the best way to handle heterogeneity in the meta-analysis of genetic studies. 
Our real data example is based on 'pseudo multi-cohorts' that each have much smaller sample sizes and thus much less power in the variable selection step than an analysis that would use the full FHS dataset. The small sample sizes may explain the discrepancy between the meta-analysis results and Procedure B, which has been shown in our simulation study to perform closest to a combined analysis.

We expect that the conclusions presented in this article are relevant to a variety of other Lasso-based methods. Because we did not use any exclusive features of the particular penalized regression approach implemented in Mendel when developing the meta-analysis strategies, our methods are generally applicable to other penalized regression algorithm. We have also examined in simulations the performance of the four procedures using a pe- nalized regression that considers interaction terms and incorporates network information [23]. The results are consistent with the simulation results presented in this article.

\section{Acknowledgement}

This work was supported in part by National Institute Health grants DK078616, ES020827, GM078987, AG028321, N01 HC25195 and P01 AI050516. This research was conducted using the Linux Clusters for Genetic Analysis (LinGA) computing resources at Boston University Medical Campus.

\section{Disclosure Statement}

The authors have no conflicts of interest.

\section{References}

1 Lin DY, Zeng D: Meta-analysis of genomewide association studies: no efficiency gain in using individual participant data. Genet Epidemiol 2010;34:60-66.

2 Fisher RA: Statistical Methods for Research Workers. Edinburgh, Oliver and Boyd, 1925.

3 Mosteller F, Fisher RA: Questions and answers: question 14. Am Stat 1948;2:30-31.

4 Stouffer SA, et al: The American Soldier, vol. 1: Adjustment during Army Life. Princeton, Princeton University Press, 1949.

5 William GC: The combination of estimates from different experiments. Biometrics 1954; 10:101-129.

6 Willer CJ, Li Y, Abecasis GR: METAL: fast and efficient meta-analysis of genomewide association scans. Bioinformatics 2010;26: 2190-2191.

7 Lu C, Latourelle J, O’Connor GT, Dupuis J, Kolaczyk ED: Network-guided sparse regression modeling for detection of gene-by-gene interactions. Bioinformatics 2013;29:12411249.

8 Wu TT, Chen YF, Hastie T, Sobel E, Lange K: Genome-wide association analysis by lasso penalized logistic regression. Bioinformatics 2009;25:714-721.
9 Meinshausen N, Meier L, Buhlmann P: p values for high-dimensional regression. J Am Stat Assoc 2009;104:1671-1681.

10 Wasserman L, Roeder K: High dimensional variable selection. Ann Stat 2009;37:21782201.

11 Tibshirani R: Regression shrinkage and selection via the Lasso. J R Stat Soc Series B Stat Methodol 1996;58:267-288.

12 Candes E, Tao T: The Dantzig selector: statistical estimation when $p$ is much larger than $n$. Ann Stat 2007;35:2313-2351.

13 Fan JQ, Li RZ: Variable selection via nonconcave penalized likelihood and its oracle properties. J Am Stat Assoc 2001;96:1348-1360.

14 Meinshausen N: Relaxed lasso. Comput Stat Data Anal 2007;52:374-393.

15 Radchenko P, James GM: Variable selection using adaptive nonlinear interaction structures in high dimensions. J Am Stat Assoc 2010;105:1541-1553.

16 Yuan M, Lin Y: Model selection and estimation in regression with grouped variables. J R Stat Soc Series B Stat Methodol 2006;68:4967.
17 Zou H: The adaptive lasso and its oracle properties. J Am Stat Assoc 2006;101:1418-1429.

18 Zou H, Hastie T: Regularization and variable selection via the elastic net. J R Stat Soc Series B Stat Methodol 2005;67:301-320.

19 Lange K, Papp JC, Sinsheimer JS, Sripracha R, Zhou H, Sobel EM: Mendel: the Swiss army knife of genetic analysis programs. Bioinformatics 2013;29:1568-1570.

20 Granada M, Wilk JB, Tuzova M, Strachan DP, Weidinger S, Albrecht E, et al: A genomewide association study of plasma total IgE concentrations in the Framingham Heart Study. J Allergy Clin Immunol 2012;129:840845.e21.

21 Li Y, Willer CJ, Ding J, Scheet P, Abecasis GR: $\mathrm{MaCH}$ : using sequence and genotype data to estimate haplotypes and unobserved genotypes. Genet Epidemiol 2010;34:816-834.

22 Morris AP: Transethnic meta-analysis of genomewide association studies. Genet Epidemiol 2011;35:809-822.

23 Lu C: New Approaches to Identify Gene-byGene Interactions in Genome Wide Association Studies; dissertation, Boston University, Boston, 2013. ISBN 978-1-3039-8924-7.
Meta-Analysis for Penalized Regression Methods with Multi-Cohort GWAS 